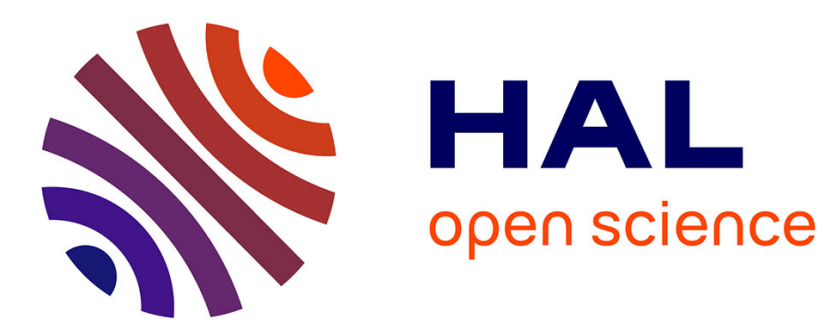

\title{
RENORMALIZED RESPONSE THEORY WITH APPLICATIONS TO PHONON ANOMALIES
}

\author{
C. Falter, Wolfgang Ludwig, M. Selmke
}

\section{To cite this version:}

C. Falter, Wolfgang Ludwig, M. Selmke. RENORMALIZED RESPONSE THEORY WITH APPLICATIONS TO PHONON ANOMALIES. Journal de Physique Colloques, 1981, 42 (C6), pp.C6-516C6-518. 10.1051/jphyscol:19816150 . jpa-00221216

\section{HAL Id: jpa-00221216 https://hal.science/jpa-00221216}

Submitted on 1 Jan 1981

HAL is a multi-disciplinary open access archive for the deposit and dissemination of scientific research documents, whether they are published or not. The documents may come from teaching and research institutions in France or abroad, or from public or private research centers.
L'archive ouverte pluridisciplinaire HAL, est destinée au dépôt et à la diffusion de documents scientifiques de niveau recherche, publiés ou non, émanant des établissements d'enseignement et de recherche français ou étrangers, des laboratoires publics ou privés. 
JOURNAL DE PHYSTQUE

Colzoque C6, supplément au $n^{\circ} 12$, Tome 42 , décembre 1981

page $\quad \mathrm{C} 6-516$

\title{
RENORMALIZED RESPONSE THEORY WITH APPLICATIONS TO PHONON ANOMALIES
}

\author{
C. Falter, W. Ludwig and M. Selmke
}

Institut für Theoretische Physik II der Universität Muenster, Domagkstr. 75, D-4400 Mienster, F.R.G.

\begin{abstract}
A recently developed renormalization method for the electron response problem in effective ion-interactions is applied to phonon anomalies. A microscopic mechanism for the anomalies is proposed and its relation to the electron-phonon-coupling parameter $\lambda$ and thus to $T_{c}$ is discussed.
\end{abstract}

The renormalization method proposed in $[1,2]$ for various applications allows for a division of the electronic density response function $D$ into a part $\bar{D}$, which renormalizes the potential (and other quantities) thus leading to screened interactions and a part which acts in the renormalized system containing the relevant degrees of freedom in a special situation. This method is able to isolate certain features of the electronic structure like the Fermi surface effect in metallic compounds, the local field effect in covalent crystals or special many body effects. The characteristic items of a definite phenomenon are prepared by decomposing the polarizability function $\pi$, which is defined in terms of quasiparticles, into a part $\bar{\pi}$ describing the screening (renormalization) and a complementary part $\Delta$, containing the relevant aspects of the response in a special case:$$
\pi=\bar{\pi}+\Delta \text {. }
$$

This division can be shown to achieve a renormalization of $\mathrm{D}$, which is related to the interactions of bare particles. With Coulomb-interaction $v$ and dielectric function $\varepsilon$ we obtain

$$
\begin{aligned}
& \mathrm{D}=\overline{\mathrm{D}}+\left(\bar{\varepsilon}^{-1}\right)^{+} \mathrm{D}_{r^{\varepsilon^{-1}}} ; \quad \varepsilon^{-1}:=\varepsilon_{r}^{-1} \cdot \bar{\varepsilon}^{-1} ; \quad \bar{D}=\pi \bar{\varepsilon}^{-1} \\
& \mathrm{D}_{\mathrm{r}}=\Delta \varepsilon_{\mathrm{r}}^{-1} ; \quad \varepsilon_{\mathrm{r}}^{-1}=(1+\overline{\mathrm{v}} \Delta)^{-1} ; \quad \bar{\varepsilon}=1+\mathrm{v} \bar{\pi} ; \quad \overrightarrow{\mathrm{v}}^{-1}=\bar{\varepsilon}^{-1} \mathrm{v} \text {. } \\
& \mathrm{D} \cong \mathrm{D}+\left(\bar{\varepsilon}^{-1}\right)^{+} \Delta \bar{\varepsilon}^{-1} \text {. }
\end{aligned}
$$

The effective ion-interaction between two ions at $\vec{A} \neq \vec{B}[1,2]$ is given by

$$
\Phi(\vec{A}, \vec{B})=V^{I I}(\vec{A}, \vec{B})-V_{\vec{A}} D V_{\vec{B}}
$$

where $\mathrm{V}^{I I}$ is the direct ion-ion-interaction and $\mathrm{V}_{\vec{A}}$ the ion-(pseudo-) potential; $\vec{A}=(\vec{a}, \alpha)$ contains indices for unit cell and basis, resp.. The Fourier-transformed effective interaction splits according to $(4,2 \mathrm{a})$ into additive parts, the anomalous one being $[3] \quad\left(Q^{\prime}=\vec{q}+\vec{G}^{\prime} ; Q^{\prime \prime}=\vec{q}+\vec{G}^{\prime \prime}\right.$ etc. $)$

$$
\Phi^{r} \begin{array}{ll}
\alpha & \beta \\
Q, Q^{\prime}
\end{array}=-V_{\alpha}(Q)\left(\bar{\varepsilon}^{-1}\right)^{+}{ }_{Q, Q^{\prime \prime}}{ }^{D_{r Q}} Q^{\prime \prime} Q^{\prime \prime}\left(\bar{\varepsilon}^{-1}\right)_{Q^{\prime \prime \prime}} Q^{\prime} V_{\beta}\left(Q^{\prime}\right),
$$

so that the renormalized part of the dynamical matrix can be written as $\left(V_{z}\right.$ : volume of unit cell; $\vec{\xi}$ : reciprocal lattice vector)

$$
t_{i j}^{r \alpha \beta}(\vec{q})=\left(M_{\alpha M_{\beta}}\right)^{-1 / 2}\left\{\Lambda_{i j}^{r \alpha \beta}(\vec{q})-\delta_{\alpha \beta} \sum_{\gamma} \Lambda_{i j}^{r \alpha \gamma}(\vec{o})\right\}
$$


with

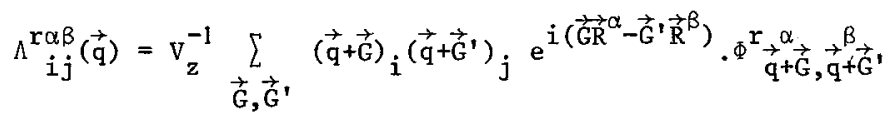

The problem of phonon anomalies and especially the correlation of soft-phonon modes to high $\mathrm{T}_{\mathrm{c}}$ is often dealt with but it is not solved in a unique way (see [4] for a review). In $[3,5,6]$ a general explanation for the phonon anomalies is proposed. As a minimal assumption for the use of the renormalization method in $\Delta$ we separate off the Fermi surface effect as the relevant part of the metallic binding component which we assume to be dominant in screening. The only characteristic property of a special material which enters the theory are the small critical wave vectors $\vec{q}_{c r}$ (nesting vectors), which occur in Fermi surfaces with a special geometry (high density of states at the Fermi level $\left.\varepsilon_{F},[7]\right)$. We define $\Delta$ (eq.(1)) to be the difference in the polarizabilities of the anomalous and the normal system (e.g. TaC and HfC), one having Fermi energy $\varepsilon_{F}+\delta \varepsilon$, the other one $\varepsilon_{F}: \quad \Delta=\pi\left(\varepsilon_{F}+\delta \varepsilon\right)-\pi\left(\varepsilon_{F}\right)$. $\Delta$ contains all the effects originating from the different topologies of the Fermi surfaces in the two materials. In our model calculations $[3,5,6]$ we use eq. (5) with a diagonal renormalization for the potentials and obtain, Iinear in $\Delta$,

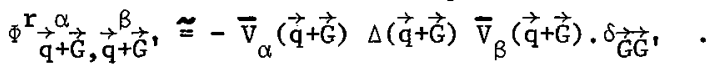

$\Delta$ can be shown to be essentially the difference of the free electron polarizabilities $\pi_{F E}$

$$
\Delta \equiv \pi_{F E}\left(\varepsilon_{F}+\delta \varepsilon\right)-\pi_{F E}\left(\varepsilon_{F}\right) \cong \frac{\partial}{\partial k_{F}}\left(\pi_{F E}\right) \cdot \delta k=\frac{\delta k}{|\vec{q}+\vec{G}|} z\left(\varepsilon_{F}\right) \cdot \ln \left|\frac{2 k_{F}+|\vec{q}+\vec{G}|}{2 k_{F}-|\vec{q}+\vec{G}|}\right| .
$$

In general $\Delta$ will have a pronounced $\vec{q}$-space resonance structure at the $\vec{q}_{c r}$ (or at $2 \mathrm{k}_{\mathrm{F}}$ in the mode1, resp.) of the Fermi surface (Fig. 1). This resonance then occurs in the effective ion-interaction (9) and finally in the dynamical matrix $(6,7)$. The magnitude of the anomaly effect is determined by the competition between the strength of the renormalized electron-ion-interaction and the density of states $z\left(\varepsilon_{F}\right)$, eqs. $(9,10)$. If the resonance condition $|\vec{q}+\vec{G}| \approx 2 k_{F}$ is satisfied for $\vec{G}=0$ (i.e. small

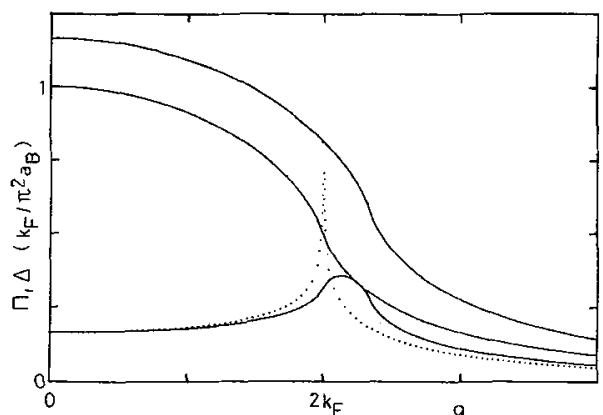

1. Exact value for $\Delta$ together with the corresponding free electron polarizabilities (full lines) and estimated value according to eq.(10) (dotted line).

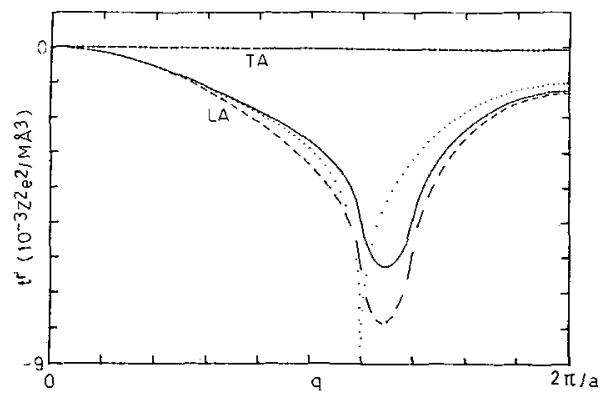

2 Anomalous part of the dynamical matrix $t$ for the $(1,0,0)$-direction of cubic symmetry. $t^{r}$ is shown for the exact $\Delta$ (full 1ine), the estimated $\Delta$ (dotted line) and with Hubbard-correction for exchange and correlation in $\Delta$ and $\vec{\varepsilon}^{-1}$ (dashed line). 
critica1 wave vectors), we have strong potentials $\bar{v}_{\alpha}[3,5,6]$ and thus an observable anomaly effect if $\mathrm{Z}\left(\varepsilon_{F}\right)$ is high (Fig.2). The corresponding frequency shift is $\Delta \omega \cong$ $t^{r} / 2 \bar{\omega}$, $\bar{w}$ being the frequency of the reference system. The factor $\vec{q}_{i} \cdot \vec{q}_{j}$ in (7) tells us, that the anomaly only occurs in LA-modes. In the case of a second sublattice (NaC1-structure) there is a coupling between LA/LO- and TA/TO-modes; then the frequency-shift in $(1,0,0)$ is approximately (linearly in $\mathrm{t}^{\mathrm{r}}$ ) given by

$$
\Delta \omega_{ \pm} \cong\left(4 \bar{\omega}_{ \pm}\right)^{-1}\left\{t_{M M}^{r}+t_{C C}^{r} \pm\left[\gamma_{1}\left(t_{M M}^{r}-t_{C C}^{r}\right)+\gamma_{2} t_{M C}^{r}\right]\right\},
$$

where $t^{r}$ denotes the anomalous components of the dynamical matrix (6), M stands for metal, $C$ for carbon, resp. and \pm denote optical or acoustical modes, resp.. Eq. (11) shows the scaling of $\Delta \omega_{ \pm}$with the inverse frequency $\bar{\omega}_{ \pm}$of the reference system; this explains the suppression of the anomaly in the optical modes relatively to the acoustic ones.

We will now trace how the anomalies due to the Fermi surface effect enter the electron-phonon-coupling parameter $\lambda$ and thus the critical temperature $T_{c}$ of a superconductor. According to [4] the (isotropically) averaged expression for $\lambda$ is $\lambda=\sum_{\alpha} \mathrm{z}\left(\varepsilon_{\mathrm{F}}\right) \frac{\left\langle\mathrm{J}_{\alpha}^{2}\right\rangle}{M_{\alpha}\left\langle\omega^{2}\right\rangle} ;\left\langle\mathrm{J}_{\alpha}^{2}\right\rangle=\left\langle\left|\left\langle\overrightarrow{\mathrm{k}}\left|\frac{\partial}{\partial \overrightarrow{\mathrm{R}}} \mathrm{v}_{\alpha}^{\mathrm{SC}}\left(\overrightarrow{\mathrm{r}}-\overrightarrow{\mathrm{R}}_{\alpha}\right)\right| \overrightarrow{\mathrm{k}}^{\prime}\right\rangle\right|^{2}\right\rangle_{\mathrm{FS}} ;\left(\mathrm{T}_{\mathrm{C}}\right)_{\mathrm{BCS}} \approx \theta_{\mathrm{D}} \exp (-1 / \lambda) .(12)$ The gradient of the total self-consistent crystal potential $\mathrm{V}_{\alpha}^{\mathrm{SC}}$ (harmonic theory) in (12) can be calculated in linear response theory. Thus the sane effects which produce the phonon anomalies enter $\left\langle\mathrm{J}_{\alpha}^{2}\right\rangle$ quadratically via $\Delta$, eq. (8); the anomalous contribution is given by $[3,6] \quad \varepsilon^{-1} v_{\alpha}=\varepsilon_{r}^{-1}\left(\bar{\varepsilon}^{-1} v_{\alpha}\right) \cong(1-\bar{v} \Delta) \bar{v}_{\alpha}$.

This indicates clearly, that the squared matrix element $\left\langle\mathrm{J}_{\alpha}^{2}\right\rangle$ in (12) is reduced in general compared to the reference system. On the other hand the high $Z\left(\varepsilon_{F}\right)$-values of the special Fermi-surfaces under consideration produce an increase in $\lambda$. Thus the product $Z\left(\varepsilon_{F}\right),\left\langle J_{\alpha}^{2}>\right.$ to a certain extent is limited or at least, it should vary less than either $\mathrm{Z}\left(\varepsilon_{\mathbf{F}}\right)$ or $\left\langle\mathrm{J}_{\alpha}^{2}\right\rangle$; but $\left\langle\omega^{2}\right\rangle$ 1ikewise involves the electron-phonon-coupling and varies similarly as $\left\langle J_{\alpha}^{2}\right\rangle$ (phonon-softening), so that we can expect an approximative proportionality between $\lambda$ and $\mathrm{Z}\left(\varepsilon_{\mathrm{F}}\right)$. These theoretical predictions are supported empirically on the basis of experimental data for the transition metals and compounds $[8]$.

References. - 1.C.Falter and M.Selmke, Phys.Rev. B21, 2078(1980); 2.C.Falter, W.Ludwig and M.Selmke, Phys.Lett. 82A, 195(1981); 3.C.Falter, Habil.Schrift (to be published); 4.S.K.Sinha, P.B.Allen, Dynamical Properties of Solids $\underline{3}$, North Holland, Amsterdam (1980); 5.C.Falter and M.Selmke, Phys.Rev. B24 (to appear 1981); 6.C.Falter, W.Ludwig and M.Selmke, Phys.Lett. A (to be pub1ished); 7.B.M.Klein, L.L.Boyer and D.A.Papaconstantopoulos, Solid State Comm. 20, $937(1976)$; 8. C.M.Varma and W. Weber, Phys.Rev. B19, 6142(1979). 